\title{
Primary Extraskeletal Ewings Sarcoma of Ala of Nose.
}

\author{
${ }^{1}$ Dr. Irappa Madabhavi , ${ }^{2}$ Dr. Apurva Patel,${ }^{3}$ Dr. Mukesh Choudhary, ${ }^{4}$ Dr. Asha \\ Anand, ${ }^{5}$ Dr. Sandip Shah, ${ }^{5}$ Dr. Suhas Aagre, ${ }^{7}$ Dr. Trupti R Jansari, ${ }^{8}$ Dr. \\ Vishalkumar Bhardava \\ ${ }^{1,2,3,4,5,6}$ Department of Medical and Paediatric Oncology, GCRI, Ahmedabad, Gujarat, India. \\ ${ }^{7}$ Department of Pathology, GCRI, Ahmedabad, Gujarat, India. \\ ${ }^{8}$ Department of Radiology, GCRI, Ahmedabad, Gujarat, India.
}

\begin{abstract}
Extra skeletal Ewing's sarcoma is often described as a tumour involving the soft tissues of the lower extremities and the para vertebral region. Ewing's sarcoma presents a rare tumour of the head and neck, and even rarer in the nasal cavity and/or paranasal sinuses. Primary Ewing's sarcoma of the ala of the nose has not been commonly described previously in the English literature. We present a case of primary Ewing's sarcoma of the nose in a 23-year-old male, presented as a fleshy mass in the ala of the left side of the nose with symptoms of nasal obstruction. The obstructing lesion was excised and microscopy showed a neoplasm composed of comparatively uniform undifferentiated cells forming solid nests. The cytoplasm of the cells was clear but poorly demarcated, partly vacuolated and contained much glycogen. The cells expressed the MIC2 gene (using the CD99 marker). Managed with wide local excision and all resection margins are microscopically free of tumour margins. Post-operative adjuvant chemotherapy was started with High-Risk protocol (vincristine, doxorubicin, cyclophosphamide and mesna alternating every 3 weeks combined ifosfamide and etoposide) and is under follow up. An extensive review of literature, to the best of our knowledge, did not reveal many cases of EES of ala of nose.
\end{abstract}

Keywords: Ewing's sarcoma, extra skeletal, nasal cavity, small round cell tumour

\section{Introduction}

Ewing's sarcoma accounts for approximately $10 \%$ of all primary bone tumours. Ewing's sarcoma most commonly occurs in the long bones and pelvis. The diaphysis is often involved when it occurs in the long bones. There is a male preponderance of approximately 1.6: $1^{1}$. Extra skeletal Ewing's sarcoma (EES) is an uncommon, rapidly growing, and round-cell malignancy of uncharacterized mesenchymal cell origin. The extra skeletal forms usually occur in the soft tissue of lower extremities, para vertebral tissues, chest wall, retroperitoneal and rarely in the head and neck region ${ }^{1}$. Primary Ewing's sarcoma of the head and neck is rare condition, accounting for 2-3\% ${ }^{2}$. Only few case reports have been published in world literature. Tefft et al first described EES in 1969 to be histologically similar to primary Ewing's sarcoma of bone ${ }^{3}$. EES is a rare soft tissue neoplasm's, which can develop in the soft tissues at any location. It occurs predominantly in adolescents and young adults between the ages of 10 and 30 years and it follows an aggressive course with a high rate of recurrence. Although ES is extremely rare among nasal cavity neoplasms, it should be considered in the differential diagnosis.

\section{Case Report}

A 23-year-old male visited the otorhinolaryngology department for nasal obstruction, epistaxis, headache and intermittent rhinorrhea for 2 months. Nasal examination revealed a reddish, erythematous, fleshy firm to hard mass in the ala of the left nasal cavity (Figure.1).

The obstructing lesion was excised and microscopy showed a neoplasm composed of comparatively uniform, small, round or oval undifferentiated cells forming solid nests. The cytoplasm of the cells was clear but poorly demarcated, partly vacuolated and contained much glycogen (Figure. 2). Immunohistochemistry showed positivity for CD-99 (Figure 5), CK, S-100, EMA and Vimentin but showed negative reaction for leukocyte common antigen, Synaptophysin and Neuron specific enolase.

Preoperative MRI revealed T1W-Fat suppressed imag showing well defined heterogeneously hypo intense lesion (Figure. 3) \& heterogeneously hyper intense lesion on T2W image (Figure. 4) in ala of the left side of the nose. Postoperative whole body Technetium-99 PET scintigraph shows no lesion related to Ewing's Sarcoma (Figure. 6). For this reason patient was accepted as having a Primary extra skeletal Ewing's sarcoma of ala of nose.

All the routine biochemical like Kidney function tests, Liver function tests and haematological investigations were within the normal range. Bone marrow aspiration and biopsy was not involved by malignancy. 
Post-operative adjuvant chemotherapy was started with High-Risk protocol (vincristine, doxorubicin, cyclophosphamide and mesna alternating every 3 weeks combined ifosfamide and etoposide). Chemotherapy was given for 17 cycles and was uneventful. Patient is under surveillance for any recurrence or progression of the disease at local site or metastasis.

\section{Discussion:}

Ewing's sarcoma is distinguished by two types: skeletal type and extra skeletal type. Most commonly, Ewing's sarcoma arises from skeletal structures, especially long bones (35\%), and pelvis (24\%). Extra skeletal Ewing's sarcoma (EES) is an uncommon, rapidly growing, round-cell malignancy of uncharacterized mesenchymal cell origin. The extra skeletal forms usually occur in the soft tissue of lower extremities, para vertebral tissues, chest wall, retroperitonium and rarely in the head and neck region ${ }^{1}$. Primary Ewing's sarcoma of the head and neck is rare condition, accounting for $2-3 \%{ }^{2}$.

Clinically, patients with EES usually present with a painless rapidly growing mass. Our patient presented with a rapidly growing mass in the ala of the left side of the nose with nasal obstruction, epistaxis, headache and intermittent rhinorrhea for 2 months. Computed tomography scan may show a low attenuation mass with heterogeneous enhancement with contrast administration. MRI findings include signal iso-intense to muscle on T1-W images and hyper intense on $\mathrm{T} 2-\mathrm{W}$ images with heterogeneous enhancement after gadolinium administration ${ }^{4}$.

The histological image of EES is a small, blue, round cell with a scanty cytoplasm and it is often confused with other small round cell tumours, but recent advances in immunohistochemistry have helped in diagnosis of ESS. This family of tumours shares common cytogenetic and molecular changes which involve of $\mathrm{t}$ $(11 ; 22)$ in $90 \%$ cases. The tumours also share expression of glycoprotein surface antigen (MIC2) CD99, a cell membrane protein of unknown function. ${ }^{5}$

Diagnostic staging at presentation must include appropriate search and staging for metastases, which are detected in about $25 \%$ of patients. The most common metastatic sites are the lungs and the pleural space, the skeletal system, and the bone marrow, or combinations thereof. Fluorine-18 fluorodeoxyglucose positron emission tomography (FDG-PET) has recently been proven to be a highly sensitive screening method for the detection of bone metastases in Ewing's sarcoma. In detecting bone metastases, FDG-PET may be even more sensitive than whole-body MRI scans ${ }^{6}$.

Early awareness and wide resection followed by chemotherapy and/or radiotherapy might improve the long term survival of patients with EES. Chemotherapy reduces the tumour size and clear micro metastasis which is present in $80 \%$ of cases. The chemotherapy protocol scheme consisted vincristine $2 \mathrm{mg} / \mathrm{m},{ }^{2}$ doxorubicin $75 \mathrm{mg} / \mathrm{m}^{2}$, cyclophosphamide $1,200 \mathrm{mg} / \mathrm{m}^{2}$ and MESNA alternating every 3 weeks with combined ifosfamide $1,800 \mathrm{mg} / \mathrm{m}^{2} / \mathrm{d}$ for 5 days and etoposide $100 \mathrm{mg} / \mathrm{m}^{2} / \mathrm{d}$ for 5 days. ${ }^{7}$

The prognosis for Ewing's sarcoma depends on the presence of metastases, because Ewing's sarcoma is highly malignant and metastasizes early to bones and lungs. Five year survival rates of EES according to various reports have been anywhere between 38 and 67\%. Although the prognosis for this tumour is poor, an early and adequate surgical resection followed by adjunctive chemotherapy and radiotherapy for microscopically positive surgical margins improve the survival rate ${ }^{8,9}$.

We hereby conclude that although wide spectrum of nasal small round cell tumours cause significant diagnostic challenges, careful evaluation and sound knowledge of light microscopic features with the help of ancillary techniques like immunohistochemistry and cytogenetic will help for accurate diagnosis and appropriate management of the patient. 


\section{Figures}

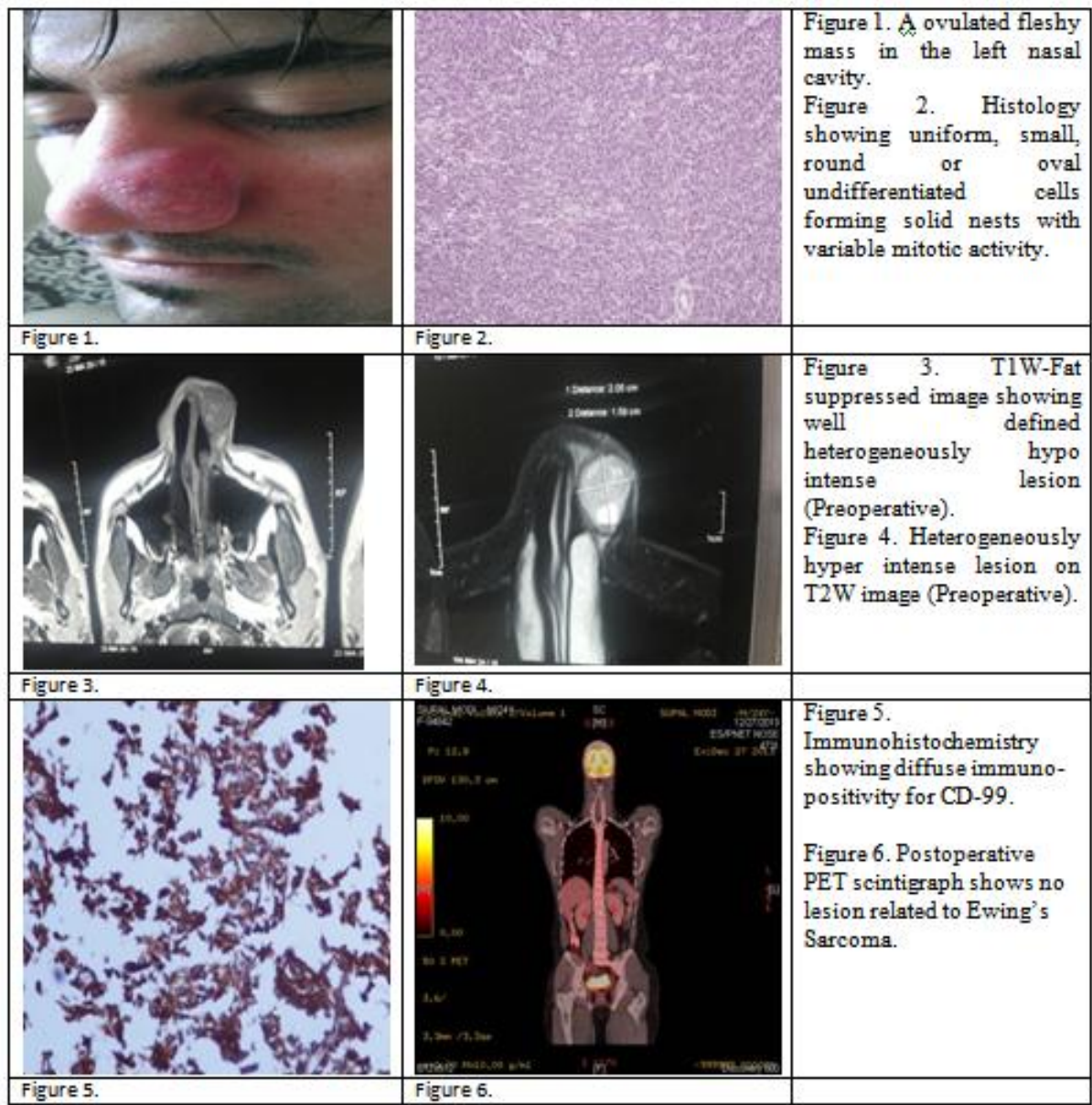

\section{References}

[1]. Iriz A, Albayrak L, Eryilmaz A. Extra skeletal primary Ewing's sarcoma of the nasal cavity. Int J Pediatr Otorhinolaryngol 2007; 2:194-7.

[2]. Dahlin, D.C. (1978): Bone tumours, General aspects and data on 6,221 cases. Third Edition, Springfield, Illinois Charles C Thomas, 274-287.

[3]. Tefft M, Vawter GF, Mitus A. Para vertebral 'round cell' tumours in children. Radiology 1969; 92(7):1501-09.

[4]. O’ Keeffe F, Lorigan JG, Wallace S. Radiological features of extra skeletal Ewing's Sarcoma. Br J Radiol 1990; 63(750): 456-60.

[5]. de Alava E, Kawai A, Healey JH, Fligman I, Meyers PA, Huvos Ag, et al. EWS-FLI1 fusion transcript structure is an independent determinant of prognosis in Ewing's Sarcoma. J Clin Oncol 1998 Aug; 16(8):2895.

[6]. Daldrup-Link HE, Franzius C, Link TM et al. Whole-body MR imaging for detection of bone metastases in children and young adults: comparison with skeletal scintigraphy and FDG PET. AJR Am J Roentgenol2001; 177:229-236.

[7]. James S. Miser, et al. Treatment of Metastatic Ewing's Sarcoma or Primitive Neuroectodermal Tumour of Bone: Evaluation of Combination Ifosfamide and Etoposide-A Children's Cancer Group and Paediatric Oncology Group Study: J Clin Oncol 22:28732876.

[8]. Rud NP, Reiman HM, Pritchard DJ, Frassica FJ, Smithson WA. Extra osseous Ewing’s Sarcoma: A study of 42 cases. Cancer 1989; 64(7): 1548-53.

[9]. Raney RB, Asmar L, Newton WA Jr, et al. Ewing's sarcoma of soft tissues in childhood: A report from the intergroup rhabdomyosarcoma study 1972-1991. J Clin Oncol 1997; 15(2):574-82. 